\title{
Extended thermodynamics, effective elastic coefficients and electromagnetoelastic waves in superconducting layer
}

Received: 22 July 2017 / Accepted: 22 November 2017 / Published online: 7 December 2017

(C) The Author(s) 2017. This article is an open access publication

\begin{abstract}
The paper deals with the analysis of an influence of the thermal field and its relaxation properties on the compressional and flexural magnetoelastic waves propagation in a vibrating superconducting layer. The investigations have been confined only to the vortex elastic field in the type-II superconductor. The description is based on the extended thermodynamical model of interactions. Contrary to the existing dynamical descriptions of electromagnetothermoelastic interactions in solids and/or in the magnetic vortex field of elastic character in the type-II superconductor, the influence of the thermal field on the electromagnetoelastic field (or electromagnetoelastic waves in such a medium) has been considered by the use of the effective elastic coefficients dependent on temperature and the relaxation time of the thermal field in low temperatures.
\end{abstract}

Keywords Thermodynamics of superconductors · Magnetic vortex waves · Effective elasticity

\section{Introduction}

Electromagnetothermoelastic (EMTE) coupled interactions occur in many physical systems. They are observed, among others, in solids of different electromagnetic properties such as dielectric, semiconducting and superconducting ones. Particularly, if they take place in low temperatures, the most interesting are materials of superconducting properties. The external magnetic field penetrates the type-II superconductor (SC2) in the form of the Abrikosov vortices (see [2-4]). Around each of them the supercurrent flows so there exist the Lorentz force interactions among them creating an additional mechanical (elastic) field (except for the mechanical one of the superconducting material as itself). Near the lower critical magnetic field intensity limit $H_{c 1}$, that field has also viscous character. Since the superconductivity occurs in the low temperatures where the thermal field has the relaxation character, the most suitable thermodynamical model is the extended one [8-10] to describe more precisely dynamical interactions and processes in the EMTE field in the SC2 and its vortex field.

Communicated by Attila Imre.

B. T. Maruszewski · M. A. Jankowska $(\bowtie) \cdot$ R. Starosta

Faculty of Mechanical Engineering and Management, Institute of Applied Mechanics, Poznan University of Technology,

Jana Pawla II 24, 60-965 Poznan, Poland

E-mail: malgorzata.jankowska@put.poznan.pl

B. T. Maruszewski

E-mail: bogdan.maruszewski@put.poznan.pl

R. Starosta

E-mail: roman.starosta@put.poznan.pl 
We have confined in the paper to the EMTE interactions within only soft vortex field in the SC2, because we have omitted pinning of vortices on defects of the crystal lattice of the superconductor. So the paper aims at determining the dispersion and amplitude distributions of the EMTE waves propagating along soft vortices in the superconducting vibrating layer (cf. $[12,17]$ ).

Contrary to the existing thermodynamical models $[19,20]$ and description of the thermal direct influences on the electromagnetoelastic field in any material and also in only superconducting vortices, the above processes have been this time analyzed with the help of the effective elastic coefficients (Young's modulus-like and Poisson's-like ratio) instead of the common constant Young's modulus and Poisson's ratio. Such effective coefficients are dependent on temperature, the thermal relaxation time and the vibrational frequency during mechanical vibrations of the considered vortex layer [13,14]. All the considerations presented in the paper have been done in the continual solid-state model for distances among vortices are in the nanoscale and the size of the layer is in the macroscale. Note that the considered problems in the paper indicate their interdisciplinary character. Therefore, the contents form an example that can be used as the application of a theoretical problem in the practice.

The famous Maugin's dictum says: There is no general agreement on their expressions as they depend either upon the taste of the scientist or on the quality and fineness of the model used to build them at a sub-macroscopic scale [17].

\section{Thermodynamical foundations}

Before we focus our attention on the main aim of the paper, i.e., on the problem of the compressional and flexural waves propagating along the vibrating layer formed solely by the Abrikosov vortices arrangement in the SC2, if their elastic features are characterized by the effective elastic coefficients, we recall the fundamental structure of the thermodynamical model of the EMTE processes running in the vortex array of the SC2 $[8,9,11,16]$.

The structure of that model consists of:

- the vector of state- the set of independent variables

$$
C=\left\{\varepsilon_{i j}, \dot{\varepsilon}_{i j}, \phi, A_{i}, T, T_{, i}, \psi, \psi^{*}, \psi_{, i}, \psi_{, i}^{*}, q_{i}, j_{i}^{S}\right\},
$$

- the fundamental laws - the balances of mass, momentum, moment of momentum and the internal energy, Maxwell's equations, the evolution equations of heat flux, superconductivity internal variable and supercurrent, i.e.,

$$
\begin{gathered}
\dot{\rho}+\rho v_{k, k}=0, \\
\rho \dot{v}_{k}-\sigma_{i k, i}-e_{i j k}\left(j_{i}^{N}+j_{i}^{S}\right) B_{j}-f_{k}=0, \\
\rho \dot{e}-\sigma_{i k} v_{k, i}-q_{k, k}-\left(j_{i}^{N}+j_{i}^{S}\right) \mathrm{E}_{i}-\rho r=0, \\
\frac{1}{\mu_{0}} A_{i, k k}-j_{i}^{N}-j_{i}^{S}=0, \\
\dot{q}_{k}-Q_{k}(C)=0, \\
\dot{\psi}-\Psi(C)=0, \\
\dot{\psi}^{*}-\Psi^{*}(C)=0, \\
\dot{j}_{k}^{S}-J_{k}^{S}(C)=0, \\
\mathrm{E}_{i}=E_{i}+e_{i j k} v_{j} B_{k}, \\
E_{i}=-\phi_{, i}-\frac{\partial A_{i}}{\partial t}, \\
B_{i}=e_{i j k} A_{k, j}, \\
B_{i}=\mu_{0} H_{i},
\end{gathered}
$$

- entropy inequality

$$
\rho \dot{S}+\Phi_{k, k}-\frac{\rho r}{T} \geq 0
$$




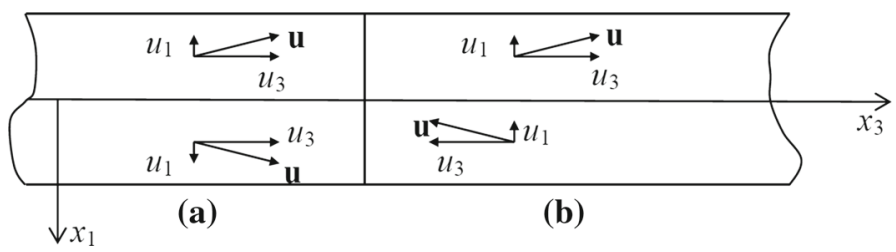

Fig. 1 a Symmetric-compressional wave C; b antisymmetric-flexural wave F

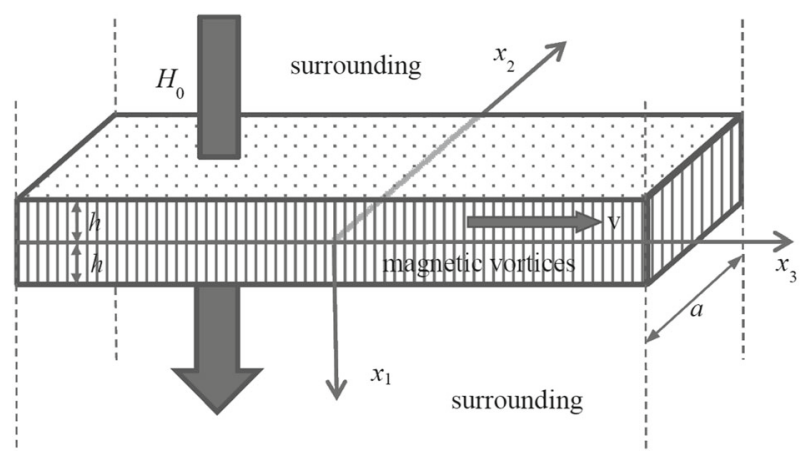

Fig. 2 Vortices in superconducting layer and geometry of the problem

- the vector of constitutive functions - the set of dependent variables

$$
Z=\left\{\sigma_{i k}, e, S, \Phi_{k}, j_{k}^{N}, Q_{k}, \Psi, \Psi^{*}, J_{i}^{S}\right\},
$$

- and the constitutive theory which determines proper relations between the vector of constitutive functions and the vector of state: the theory which consists of laws of state, laws of processes and the residual inequality (for details see $[8,9]$ ).

In the above thermodynamical model, it was assumed that the elastic strain and stress tensors are symmetric and that the vortex field has also a viscous character because inside each of the vortex core the normal Ohm'slike electric current flows.

In that model, the following notations have been used: $\varepsilon_{i j}$ is the elastic strain tensor, $\sigma_{i j}$ is the viscoelastic stress tensor, $\phi$ and $A_{i}$ are the scalar and vector electromagnetic potentials, respectively, $T$ is temperature, $q_{i}$ and $j^{S}$ are the heat flux and the supercurrent density, respectively, $\psi$ is the order parameter (the internal variable, the wave function of the Cooper pairs) and the superimposed star denotes its complex conjugate, $v_{k}$ is the velocity of the vortex field point, $\rho$ is the density of the material in the normal state as the counterpart in the mixed SC2 (i.e., the mass of the normal part of the body related to its total volume $V$ ), $j^{N}$ is the density of Ohm's-like current, $\mathrm{E}_{i}$ and $B_{i}$ are the electric field intensity and the magnetic induction in moving frames, respectively, $e$ is the internal energy density, $r$ is the heat source distribution, $S$ is the entropy density, and $\Phi_{i}$ is its flux.

\section{Wave equations}

The main aim of our considerations is the analysis of a problem of the EMTE compressional $\mathrm{C}$ and flexural $\mathrm{F}$ waves propagation (Fig. 1 [6]) along the previously defined vortex field in the type-II superconducting layer (Fig. 2).

Following the extended thermodynamical model of interactions in the magnetic vortex field presented in the previous section, the general electromagnetoelastic equations read $[12,17]$ :

$$
\begin{aligned}
& \mu u_{i, j j}+\eta \dot{u}_{i, j j}+(\lambda+\mu) u_{j, i j}+\frac{1}{3} \eta \dot{u}_{j, i j}-\mu_{0}\left(h_{r, i}-h_{i, r}\right) H_{r}^{0}-\rho \ddot{u}_{i}=0, \\
& \lambda_{0}^{2} h_{i, k k}-h_{i}+u_{i, k} H_{k}^{0}-u_{k, k} H_{i}^{0}=0, \\
& \lambda_{0}^{2} e_{i, j j}-e_{i}+\mu_{0} e_{i j k} \dot{u}_{j} H_{k}^{0}=0 .
\end{aligned}
$$


These equations have been already linearized by

$$
H_{k}=H_{k}^{0}+h_{k}, \quad\left|h_{k}\right|<<\left|H_{k}^{0}\right|
$$

and in this stage of considerations thermal influences have been omitted (the reason was mentioned before), but the viscous features of the vortex field have been taken into account. $u_{k}$ is the displacement vector of the vortex field point, $H_{k}^{0}$ is the constant part of the applied normal to the layer magnetic field, $h_{k}$ is its perturbation, $e_{k}$ is the electric field intensity in the sense of (17), $\lambda$ and $\mu$ are Lamè's coefficients, $\mu_{0}$ is the permeability of vacuum, and $\eta$ is the viscosity coefficient [4]

$$
\eta=\frac{\phi_{0} \mu_{0} H_{c 2}}{\rho_{n}} .
$$

$\phi_{0}$ denotes the magnetic flux, $H_{c 2}$ is the upper magnetic field strength limit of the $\mathrm{SC} 2, \rho_{n}$ is the resistivity of the superconducting material in the normal state, and $\lambda_{0}$ is the London penetration depth.

The solutions of equations (16) are looked for in the form

$$
f\left(x_{1}, x_{3}, t\right)=\tilde{f}\left(x_{1}\right) \exp \left[i\left(\omega t-k x_{3}\right)\right],
$$

where $\tilde{f}\left(x_{1}\right)$ are the amplitudes of signals $u_{1}, u_{2}, u_{3}, h_{1}, h_{2}, h_{3}, e_{1}, e_{2}, e_{3}$ propagating in $x_{3}$ direction with the velocity $v$ (see Fig. 2).

To facilitate analysis in the sequel, all the relations will be presented in the dimensionless form with the help of the following substitutions ( $\tilde{c}$ denotes speed of light):

$$
\begin{aligned}
x_{1} & =h x, \quad x_{2}=h y, \quad x_{3}=h z, \quad t=T \tau, \quad T=\frac{h}{v_{T}}, \quad v_{T}=\sqrt{\frac{\mu}{\rho}}, \quad \Omega=\omega T, \\
c & =\frac{k v_{T}}{\omega}=\frac{v_{T}}{v}, \quad \tilde{\lambda}_{0}=\frac{\lambda_{0}}{h}, \quad \tilde{\mu}=\frac{K}{\mu}, \quad K=\lambda+\frac{2}{3} \mu, \quad \tilde{\lambda}=\frac{\lambda}{\mu}, \quad \tilde{\rho}=\frac{\rho v_{T}^{2}}{\mu}=1, \\
H^{0} & =H_{c 1} H_{0}, \quad E=\tilde{c} \mu_{0} H_{c 1}, \quad c_{1}=\frac{v_{T}}{\tilde{c}}, \\
u_{1} & =h u_{x}, \quad u_{2}=h u_{y}, \quad u_{3}=h u_{z}, \quad e_{1}=E e_{x}, \quad e_{2}=E e_{y}, \quad e_{3}=E e_{z}, \\
\tilde{\eta} & =\eta \frac{h}{v_{T}}, \quad \bar{\mu}=\frac{\mu_{0} H_{c 1}^{2}}{\mu}, \quad h_{1}=H_{c 1} h_{x}, \quad h_{2}=H_{c 1} h_{y}, \quad h_{3}=H_{c 1} h_{z}, \quad \tilde{G}=\tilde{\mu} .
\end{aligned}
$$

\section{Compressional and flexural electromagnetothermoelastic waves}

In this section, we finally focus our attention at the analysis of propagation of $\mathrm{C}$ and $\mathrm{F}$ waves along vibrating with frequency $\tilde{\omega}$ superconducting layer of magnetic vortices (see Figs. 1,2) taking also into account an influence of the thermal field. Therefore, contrary to the existing research we have replaced Lamè's elastic constants which are based on the constant Young's modulus and constant Poisson's ratio by Lamè's-like coefficients based on the following effective Young's modulus $E$ and effective Poisson's ratio $v[13,14]$

$$
\begin{aligned}
E & =\frac{1-v^{2}}{1-v_{T}^{2}}\left[E_{T}-\left(E_{S}-E_{T}\right)\left(1+v_{T}\right) \frac{1-2 v}{1-2 v_{S}} g(\tilde{\omega})\right], \\
\left(1-v_{T}\right)\left(1-2 v_{S}\right) & =(1-v)\left(1-2 v_{S}\right)-\left(v_{S}-v_{T}\right)(1-2 v)(1-v) g(\tilde{\omega}),
\end{aligned}
$$

where

$$
g(\tilde{\omega})=\frac{\tilde{\omega}^{2}\left[1-\tau_{T}^{2}\left(\tilde{\omega}^{2}-\omega_{x}^{2}\right)\right]}{\tau_{T}^{2}\left(\omega_{x}^{2}-\tilde{\omega}^{2}\right)^{2}+\tilde{\omega}^{2}} .
$$

Index " $T$ " indicates that the values of the coefficients were measured in the isothermal conditions (low forcing frequency), whereas index " $S$ " indicates that their values were measured in the adiabatic ones (high forcing frequency). Then

$$
\omega_{x}^{2}=\frac{H k_{T}}{\tau_{T} \rho_{T} c_{\nu T}}, \quad H=\left(\frac{\pi}{a}\right)^{2} .
$$


$\tau_{T}$ is the thermal relaxation time and $k_{T}$ is the heat conduction coefficient. Relations between the isothermal and adiabatic elastic coefficients $\lambda$ and $\mu$ are the following [18] (index $K$ stands for $T$ and $S$ ):

$$
\begin{gathered}
E_{K}=\frac{\mu_{K}\left(3 \lambda_{K}+2 \mu_{K}\right)}{\lambda_{K}+\mu_{K}}, \quad v_{K}=\frac{\lambda_{K}}{2\left(\lambda_{K}+\mu_{K}\right)}, \\
\mu_{S}=\mu_{T}, \quad \lambda_{S}=\lambda_{T}+\frac{T_{0}}{c_{\nu T}}\left(3 \lambda_{T}+2 \mu_{T}\right)^{2} \alpha_{t T}^{2},
\end{gathered}
$$

where $\alpha_{t T}$ is the heat expansion coefficient, $T_{0}$ is the reference temperature and $c_{\nu T}$ is the heat capacity. This way there exists quite new possibility to consider thermal influences on the electromagnetoelastic interactions in the superconducting body. Remark that $T_{0}$ cannot exceed its critical temperature value below which the superconducting phase exists. That possibility based on Eqs. (21)-(25) allows also to take into account the thermoelastic damping phenomenon $[1,7,13,15]$ (a vibrating layer causes that its upper and lower fibers are alternatively extended and compressed; it further leads to an additional heat flux as a reason of an energy dissipation) and the thermal relaxation - the natural property occurring in low temperatures familiar to the superconducting phase.

The solutions of the C and F wave equations (16) are looked for in the form (19) if

$$
\tilde{f}\left(x_{1}\right)=\left\{u_{1}, u_{3}, h_{1}, h_{3}, e_{2}\right\},
$$

since (see Fig. 2)

$$
\mathbf{u}=\left[u_{1}, 0, u_{3}\right], \quad \mathbf{h}=\left[h_{1}, 0, h_{3}\right], \quad \mathbf{e}=\left[0, e_{2}, 0\right] .
$$

On using now (26) and (20) in (16) the dimensionless $\mathrm{F}$ and $\mathrm{C}$ wave equations read

$$
\begin{aligned}
& \left(\frac{4}{3}+\frac{1}{3} \tilde{G}\right) u_{x, x x}+\Omega^{2}\left(1-c^{2}\right) u_{x}-\frac{1}{3} i \Omega c(\tilde{G}+1) u_{z, x}=0, \\
& u_{z, x x}+\Omega^{2}\left[1-c^{2}\left(\frac{4}{3}+\frac{1}{3} \tilde{G}\right)\right] u_{z}-\frac{1}{3} i \Omega c(\tilde{G}+1) u_{x, x}+i \tilde{\mu} H_{0} \Omega c h_{x}+\tilde{\mu} H_{0} h_{z, x}=0, \\
& \tilde{\lambda}_{0}^{2} h_{x, x x}-\left(1+\tilde{\lambda}_{0}^{2} \Omega^{2} c^{2}\right) h_{x}+i \Omega c H_{0} u_{z}=0, \\
& \tilde{\lambda}_{0}^{2} h_{z, x x}-\left(1+\tilde{\lambda}_{0}^{2} \Omega^{2} c^{2}\right) h_{z}+H_{0} u_{z, x}=0, \\
& \tilde{\lambda}_{0}^{2} e_{y, x x}-\left(1+\tilde{\lambda}_{0}^{2} \Omega^{2} c^{2}\right) e_{y}-i \Omega c_{1} H_{0} u_{z}=0 .
\end{aligned}
$$

These equations have to be completed by the electromagnetic field equations in vacuum

$$
\begin{aligned}
& h_{x, x x}^{p}-\Omega^{2}\left(c^{2}-c_{1}^{2}\right) h_{x}^{p}=0, \quad h_{z, x x}^{p}-\Omega^{2}\left(c^{2}-c_{1}^{2}\right) h_{z}^{p}=0, \\
& \quad e_{y, x x}^{p}-\Omega^{2}\left(c^{2}-c_{1}^{2}\right) e_{y}^{p}=0,
\end{aligned}
$$

where the superscript $p$ denotes the values of the electric and magnetic field quantities in vacuum.

Finally, the jump and boundary conditions for the set of equations (28) and (29) at the limiting planes of the layer (upper and lower) read (see Fig. 2)

$$
\begin{gathered}
\left.\sigma_{x x}\right|_{x=-1}=\left.0 \Rightarrow \frac{d u_{x}}{d x}\right|_{x=-1}=0,\left.\quad \sigma_{z x}\right|_{x=-1}=\left.0 \Rightarrow \frac{d u_{z}}{d x}\right|_{x=-1}=0, \\
\left.\left(h_{x}=h_{x}^{p}\right)\right|_{x=-1} \text { or }\left.\left(e_{y}=e_{y}^{p}\right)\right|_{x=-1},\left.\quad\left(h_{z}=h_{z}^{p}\right)\right|_{x=-1}, \\
\left.\sigma_{x x}\right|_{x=1}=\left.0 \Rightarrow \frac{d u_{x}}{d x}\right|_{x=1}=0,\left.\quad \sigma_{z x}\right|_{x=1}=\left.0 \Rightarrow \frac{d u_{z}}{d x}\right|_{x=1}=0, \\
\left.\left(h_{x}=h_{x}^{p}\right)\right|_{x=1} \text { or }\left.\left(e_{y}=e_{y}^{p}\right)\right|_{x=1},\left.\quad\left(h_{z}=h_{z}^{p}\right)\right|_{x=1} .
\end{gathered}
$$




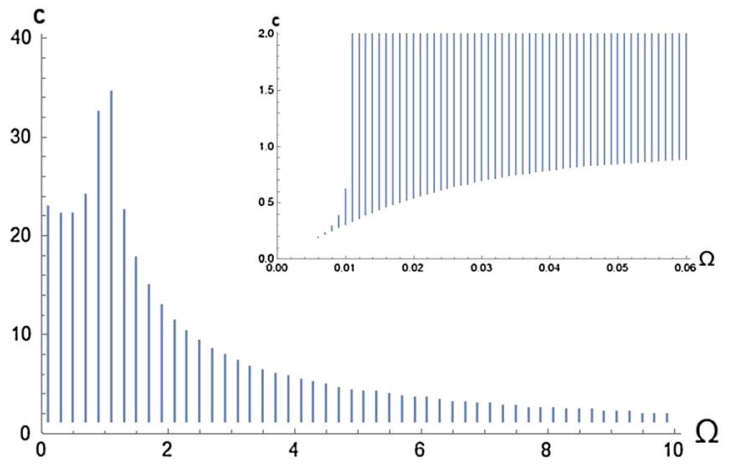

(a)

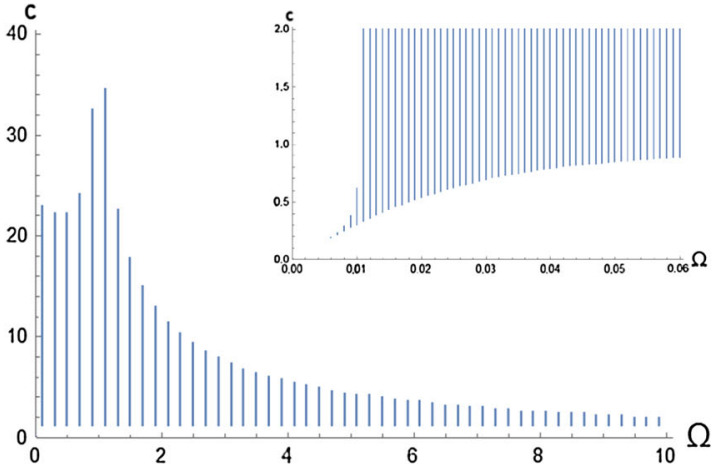

(b)

Fig. 3 Dispersion of the $\mathrm{C}$ and $\mathrm{F}$ waves in the layer if $\tilde{\omega}=0[12]$ and if $\tilde{\omega} \neq 0$ for effective $E$ and $\nu$. a $\tilde{\omega}=0, \mathbf{b} \tilde{\omega} \neq 0$

Remark, that the energy of the electromagnetic field in vacuum satisfies the radiation condition-the Poynting theorem, i.e.,

$$
\frac{d \epsilon^{p}}{d t}+s_{k, k}^{p}=0
$$

where

$$
\epsilon^{p}=\frac{1}{2}\left(\varepsilon_{0} \varepsilon_{k}^{p} \varepsilon_{k}^{p}+\mu_{0} h_{k}^{p} h_{k}^{p}\right), \quad s_{k}^{p}=e_{k i j} e_{i}^{p} h_{j}^{p},
$$

and $\epsilon^{p}$ is the electromagnetic energy, $\varepsilon_{0}$ is the permittivity of vacuum, and $s_{k}^{p}$ denotes the Poynting vector (the flux of $\epsilon^{p}$ ) (cf. [5]).

The detailed analytical forms of amplitudes (26) both in the layer and vacuum are presented in [12].

\section{Numerical results}

All the calculations conerning solutions of the problem (28)-(30) have been made for $\mathrm{YBa}_{2} \mathrm{Cu}_{3} \mathrm{O}_{6+x}(\mathrm{YBCO})$ ceramics. Since the problem has the EMTE character which concerns the solid-like state of the field of vortices, so the external magnetic field $H_{0}$ is taken slightly higher than its limiting lower value $H_{c 1}$. The results, if the layer does not vibrate (the forcing frequency $\tilde{\omega}$ is equal to zero) and all the thermal influences are completely omitted, have been presented in [12]. So, the use of the extended thermodynamical model to describe phenomena considered in that paper was not necessary because, firstly, the thermoelastic damping phenomenon did not occur and, secondly, Young's modulus and Poisson's ratio were the isothermal elastic constants —not the effective ones in described processes; also any thermal influences on the electromagnetic field did not occur. Since this paper deals with the checking of the influence of the thermal field and dynamics of the layer on the propagation of the compressional $\mathrm{C}$ and flexural $\mathrm{F}$ waves in the vortex layer firstly, to compare differences of the results presented in this paper, we show the dispersion of the $\mathrm{F}$ and $\mathrm{C}$ waves from [12].

It is seen in Fig. 3 that for the same thermal conditions the influence of the vibrations of the layer on the dispersion of $\mathrm{C}$ and $\mathrm{F}$ waves is practically negligible. However, if the forcing frequency $\tilde{\omega} \neq 0$, the extended thermodynamical model is necessary, because the interactions occur in low temperatures, the effective $E$ and $v$ are not constant, the thermoelastic damping occurs and the influence of the thermal field on the dispersion of $\mathrm{C}$ and $\mathrm{F}$ waves exists by $T_{0}, \tau_{T}$ and $c_{\nu T}$. But the reasonable influence on the dispersion of those waves is by the value of the coefficient of thermal expansion of the vortex field $\alpha_{t T}$.

Pictures presented in Fig. 4 show that the velocity of the $\mathrm{C}$ and $\mathrm{F}$ waves (see the definition of $c$ in (20)) increases if $\alpha_{t T}$ increases what confirms the fact that the superconducting properties of materials can exist only in low temperatures. It also agrees with the definition of $\alpha_{t T}$ because its value increases if temperature decreases for a material. Moreover, it is interesting that the dispersion area decreases with increasing wave velocity and if its frequency decreases. 


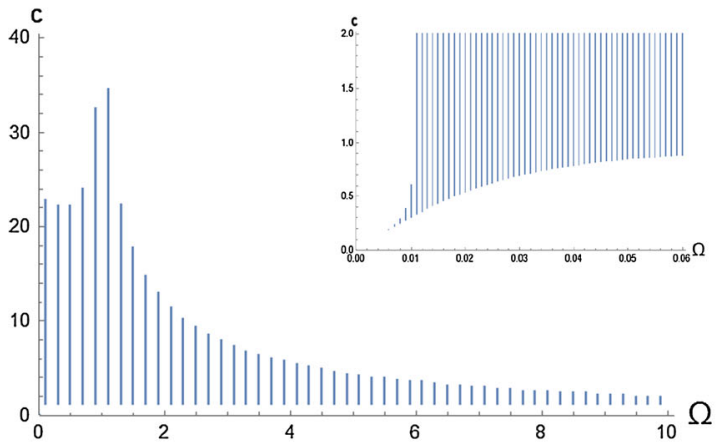

(a)

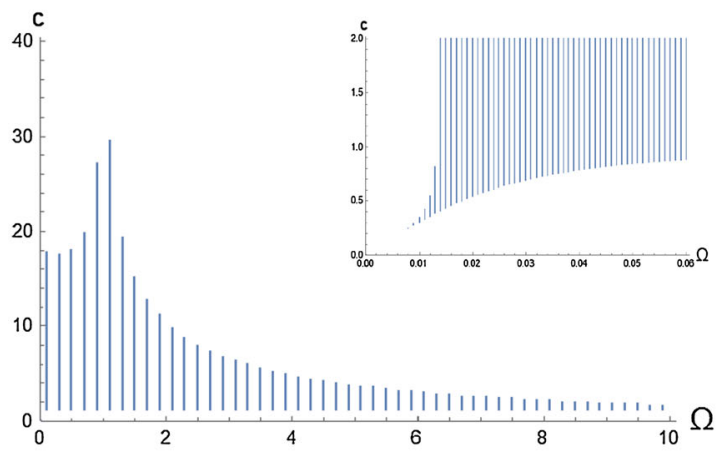

(c)

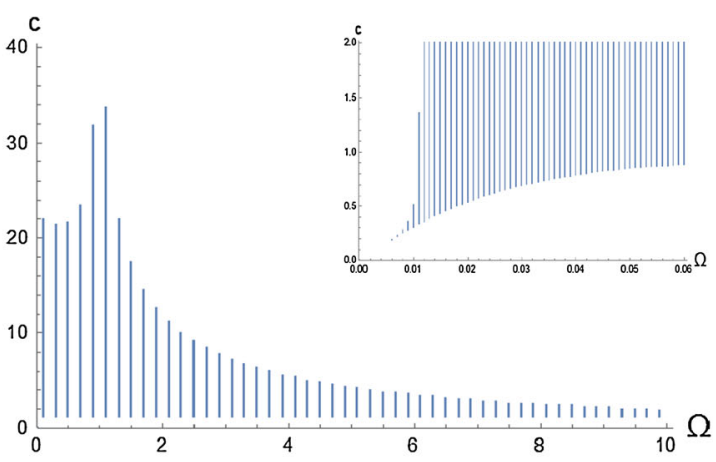

(b)

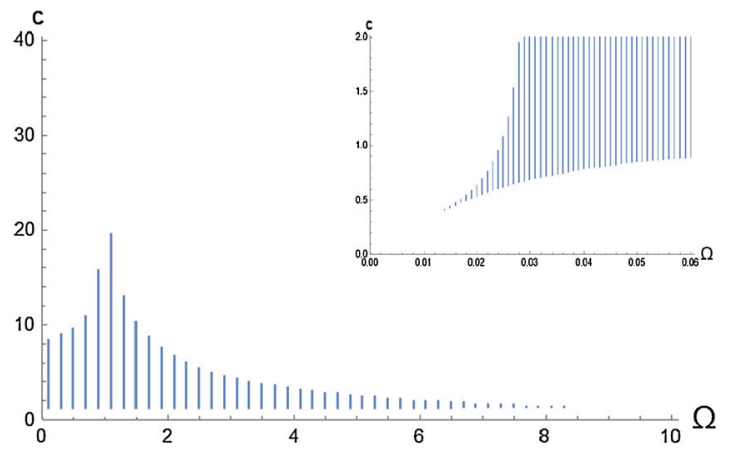

(d)

Fig. 4 Dispersion of $\mathrm{C}$ and $\mathrm{F}$ waves for $\tilde{\omega} \neq 0$ with the influence of the heat expansion coefficient $\alpha_{t T}$. $\mathbf{a} \alpha_{t T}=100$, $\mathbf{b} \alpha_{t T}=300$, c $\alpha_{t T}=700, \mathbf{d} \alpha_{t T}=1000$

\section{Conclusions}

The presented description of the EMTE interactions occurring in the SC2 vortex layer within the extended thermodynamical model in the paper was suitable. It showed that the resulting from that model field equations properly describe the compressional $\mathrm{C}$ and flexural $\mathrm{F}$ wave propagation along the vibrating layer formed solely from the magnetic vortices. Moreover, the effective elastic coefficients $E$ and $v$ better describe those interactions than the isothermal constant ones. It confirms the fact that such a choice is much better if the problem deals with a dynamics (both wave propagation and vibration phenomenon, for instance) of a medium of finite extent.

Acknowledgements The paper was supported by the Poznan University of Technology (Poland) through Grant No. 02/21/DSPB/3477.

Open Access This article is distributed under the terms of the Creative Commons Attribution 4.0 International License (http:// creativecommons.org/licenses/by/4.0/), which permits unrestricted use, distribution, and reproduction in any medium, provided you give appropriate credit to the original author(s) and the source, provide a link to the Creative Commons license, and indicate if changes were made.

\section{References}

1. Alblas, J.B.: A note of the theory of thermoelastic damping. J. Therm. Stresses 4, 333-355 (1981)

2. Blatter, G., Feigelman, M.V., Geshkenbeim, V.B., Larkin, A.L., Vinokur, V.M.: Vortices in high-temperature superconductors. Rev. Mod. Phys. 66, 1125-1388 (1994)

3. Brandt, E.H.: The flux-line lattice in superconductors. Rep. Prog. Phys. 58, 1465-1594 (1995)

4. Cyrot, M., Pavuna, D.: Introduction to superconductivity and high-T ${ }_{c}$ materials. World Scientific, Singapore (1992)

5. Eringen, A.C.: Continuum Physics, vol. III. Academic Press, London (1976)

6. Eringen, A.C., Şuhubi, E.S.: Elastodynamics, vol. II. Academic Press, New York (1975)

7. Maruszewski, B.: Nonlinear thermoelastic damping in circular plate. ZAMM 72, T75-T78 (1992) 
8. Maruszewski, B.T.: Superconducting fullerenes in a nonconventional thermodynamical model. Arch. Mech. 50, 497-508 (1998)

9. Maruszewski, B.T.: On a nonclassical thermoviscomechanical stress in the vortex field in the type-II superconductor. Phys. Stat. Sol. (b) 244, 919-927 (2007)

10. Maruszewski, B.T.: Fullerenes: thermomechanics, doping, electrical conductivity. In: Hetnarski, R.B. (ed.) Encyclopedia of Thermal Stresses, vol. 4, pp. 1831-1841. Springer, Berlin (2014)

11. Maruszewski, B.T.: Unconventional thermodynamical model of processes in material structures. In: Anders, B., Kuczma, M. (eds.) Continuous Media with Microstructure. Springer, Berlin (2015)

12. Maruszewski, B.T., Drzewiecki, A., Starosta, R.: Electromagnetoelastic waves in a vortex layer of a superconductor. J. Mech. Mater. Struct. 7, 297-307 (2012)

13. Maruszewski, B.T., Drzewiecki, A., Starosta, R.: Thermoelastic damping and thermal relaxation time in auxetics. Appl. Mech. Mater. 432, 215-220 (2013)

14. Maruszewski, B.T., Drzewiecki, A., Starosta, R.: On effective Young's modulus and Poisson's ratio of the auxetic thermoelastic material. Comput. Methods Sci. Technol. 22, 233-237 (2016)

15. Maruszewski, B.T., Drzewiecki, A., Starosta, R., Restuccia, L.: Thermoelastic damping in an auxetic rectangular plate with thermal relaxation: forced vibrations. J. Mech. Mater. Struct. 8, 403-413 (2013)

16. Maruszewski, B.T., Restuccia, L.: Mechanics of a vortex lattice in superconductors. Phenomenological approach. In: Maruszewski, B.T., Muschik, W., Radowicz, A. (eds.) Trends in Continuum Physics. World Scientific, Singapore (1999)

17. Maugin, G.A.: Non-equilibrium thermodynamics of electromagnetic solids. In: Muschik, W. (ed.) Non-equilibrium Thermodynamics with Applications to Solids, p. 214. Springer, Berlin (1993)

18. Nowacki, W.: Thermoelasticity. Pergamon, Oxford (1962)

19. van de Ven, A.A.F.: A note on 'a nonequilibrium theory of thermoelastic superconductors' by S.-A. Zhou and K. Miya. Int. J. Appl. Electromagn. Mater. 2, 169-175 (1991)

20. Zhou, S.A., Miya, K.: A nonequilibrium theory of thermoelastic superconductors. Int. J. Appl. Electromagn. Mater. 2, 21-38 (1991) 